\title{
Recent $B$ physics results from D0
}

\author{
B. Abbott $^{\mathrm{a}}$ (For the D0 collaboration)

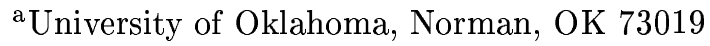

The D0 detector as collected over $3.7 \mathrm{fb}^{-1}$ of high quality data. With this large data set, D0 is able to perform a number of precision $B$ lifetime and CP asymmetry measurements. Additionally D0 is measuring a number of properties of $B$ hadrons $\left(B_{s}\right.$ and $\left.B_{c}\right)$ which are currently only accessible at the Tevatron.

\section{1. $B_{s} \rightarrow J / \psi \phi$}

The CP violating mixing phase $\phi_{s}$ is predicted to be small, $-0.04 \pm 0.01$, however, new phenomenon may modify this phase and a large value of $\phi_{s}$ may be an indication of new physics. $B_{s}$ decays are reconstructed in the mode $B_{s} \rightarrow J / \psi \phi$. Using $2.8 \mathrm{fb}^{-1}$ of data, $1967 \pm 65$ (stat) events are reconstructed. The flavor of the initial $B_{s}$ is determined by flavor tagging using both "sameside" and "opposite-side" tagging. The decay amplitude of the $B_{s}$ is separated into three independent components. These components are either longitudinal or transverse to their direction and parallel or perpendicular to each other. There is a high degree of correlation between $\Delta m_{s}, \phi_{s}$ and the two CP-conserving phases. This makes it difficult to obtain a stable fit if all parameters are allowed to vary independently. To obtain a stable fit, $\Delta m_{s}$ is fixed to the measured value of $17.77 \pm$ $0.12 \mathrm{ps}^{-1}[1]$. The two CP-conserving phases are allowed to vary around the world averages under a Gaussian constraint with width $\pi / 5$.

After applying the above constraints an unbinned likelihood fit returns $\phi_{s}=-0.57_{0.30}^{+0.24}$ and $\Delta \Gamma_{s}=0.19 \pm 0.07 \mathrm{ps}^{-1}$. Fig. 1 shows the confidence contours in the $\Delta \Gamma_{s}-\phi_{s}$ plane.

In addition, the average lifetime of the $\left(B_{s}, \bar{B}_{s}\right)$ system is measured to be $\bar{\tau}_{s}=1.52 \pm 0.05$ (stat) \pm 0.01 (syst) ps (see Fig. 2).

\section{2. $B_{s} \rightarrow D_{s}^{(*)} D_{s}^{(*)}$}

In the Standard Model, the mixing phase $\phi_{s}$ can be ignored when discussing $\Delta \Gamma_{s}$. In this case,

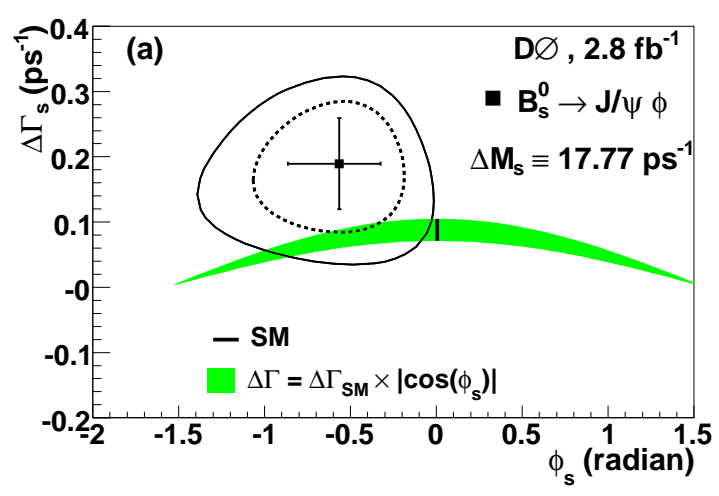

Figure 1. The confidence-level contours in the $\Delta \Gamma_{s}-\phi_{s}$ plane. The dashed curve is the $68.3 \%$ CL and the solid is the $90 \%$ CL.

the mass eigenstates are the $\mathrm{CP}$ eigenstates and any $b \rightarrow c \bar{c} s$ decay into a $\mathrm{CP}$ even ground state arises only from the CP even component. Under certain theoretical assumptions [3] the decay $B_{s} \rightarrow D_{s}^{*} D_{s}^{*}$ is CP-even to within $5 \%$ and therefore this branching ratio is related to $\Delta \Gamma_{s}$ as:

$2 B r\left(B_{s} \rightarrow D_{s}^{*} D_{s}^{*}\right) \approx \Delta \Gamma_{s}^{C P}\left[\frac{1+\cos \phi_{s}}{2 \Gamma_{L}}+\frac{1-\cos \phi_{s}}{2 \Gamma_{H}}\right]$

This analysis is based on $2.8 \mathrm{fb}^{-1}$ and studies $B_{s}$ decays into two $D_{s}^{(*)}$ mesons. The notation $D_{s}^{(*)}$ refers to either a $D_{s}$ or $D_{s}^{*}$ since it is not possible to distinguish between them due to the undetected particles in $D_{s}^{*} \rightarrow D_{s} \gamma / \pi^{0}$ decays. 


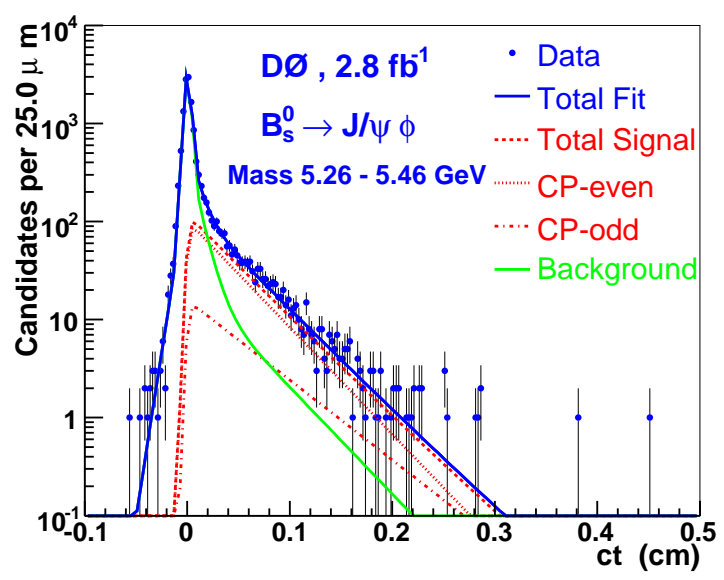

Figure 2. Lifetime distribution for $B_{s} \rightarrow J / \psi \phi$.

In this analysis, one $D_{s}^{(*)}$ decays into $\phi_{1} \pi$ and the other into $\phi_{2} \mu \nu$. The branching ratio measurement is made by normalizing the $B_{s} \rightarrow$ $D_{s}^{(*)} D_{s}^{(*)}$ decay to $B_{s} \rightarrow D_{s}^{(*)} \mu \nu$.

The correlation between $D_{s}\left(\phi_{1} \pi\right)$ candidates and $\phi_{2}(K K)$ candidates is exploited using a twodimensional unbinned likelihood fit. Fig. 3 shows the result of the fit, where the fit yields $\mathrm{N}\left(D_{s} \phi_{2} \mu\right)=31.0 \pm 9.4$. The signal fraction of $B_{s} \rightarrow D_{s}^{(*)} D_{s}^{(*)}$ is determined by assuming the background is dominated by two processes: $B^{ \pm, 0} \rightarrow D_{s}^{(*)} D_{s}^{(*)} K X$ and $B_{s} \rightarrow D_{s}^{(*)} \mu \nu \phi$. Fig 4 shows the two-dimensional mass distributions for signal and background. The plot is divided into nine regions and Monte Carlo is to determine the fraction of events in each region occupied by signal and background. The data are then fit to determine the number of signal events. The main systematic uncertainty in the measurement of the branching ratio is the uncertainties of the branching ratios found in the PDG [2]. A final branching ratio measurement of $\operatorname{Br}\left(B_{s} \rightarrow D_{s}^{(*)} D_{s}^{(*)}\right)=$ $0.042 \pm 0.015$ (stat) \pm 0.017 (syst) is obtained giving $\frac{\Delta \Gamma_{s}}{\Gamma_{s}}=0.088 \pm 0.030$ (stat) \pm 0.036 (syst).

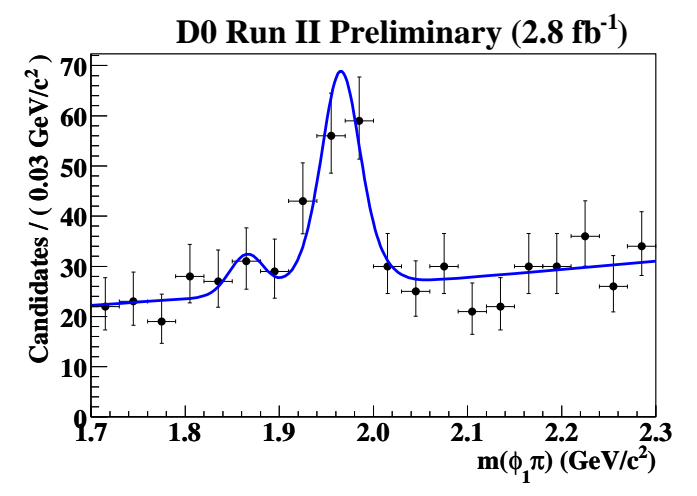

Figure 3. Invariant mass distribution $m\left(\phi_{1} \pi\right)$ for the $D_{s} \phi_{2} \mu$ sample

\section{3. $B_{c}$ mass and lifetime}

The $B_{c}$ is unique in that it is a bound state of two heavy quarks of different flavor. By studying the $B_{c}$, various theoretical models can be tested against the measured mass and lifetime. The $B_{c}$ is most easily reconstructed when it decays into a $J / \psi$ such as $B_{c} \rightarrow J / \psi \ell \nu$ (for lifetime measurements due to the high statistics) and $B_{c} \rightarrow J / \psi \pi$ (for mass measurements, since there is no undetected neutrino).

Using approximately $1.3 \mathrm{fb}^{-1}$ of data, the decay $B_{c} \rightarrow J / \psi \pi$ is reconstructed and is shown in Fig. 5. An unbinned likelihood fit is performed resulting in a signal yield of $54 \pm 12$ events, a signal significance of $5.2 \sigma$ and a $B_{c}$ mass of 6300.7 $\pm 13.6 \mathrm{MeV} / \mathrm{c}^{2}$

Using approximately $1.3 \mathrm{fb}^{-1}$ of data, the lifetime of the $B_{c}$ meson is also measured. $B_{c}$ candidates are formed by combining a $J / \psi$ with a muon. The mass of the $J / \psi+\mu$ candidate is required to be between 3 and $10 \mathrm{GeV}$. The mass can be used to separate out the signal from the five major backgrounds. A signal mass template is determined using $\mathrm{MC}$ and background templates are measured using data. Fig. 6 shows the fit to the mass distribution after subtracting the backgrounds due to $B^{+}$and the $J / \psi$ sidebands.

A fit to the data consisting of a mass shape tem- 


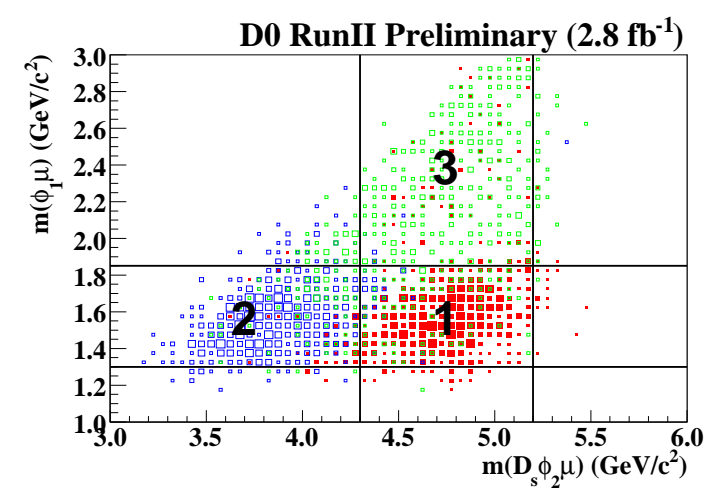

Figure 4. Two dimensional plot of $m\left(\phi_{2} \mu\right)$ versus $m\left(D_{s} \phi_{2} \mu\right)$. The signal region is dominant in region 1 while the background, $B^{ \pm, 0} \rightarrow D_{s}^{*} D_{s}^{*} K X$ is dominant in region 2 and $B_{s} \rightarrow D_{s}^{*} \mu \nu \phi$ is dominant in region 3 .

plate and a lifetime model is performed. Since the $p_{T}$ of the $B_{c}$ is not known due to the presence of the neutrino, the $p_{T}$ of the $J / \psi+\mu$ is used as an approximation. The visible proper decay length is measured and defined as $L_{x y} \frac{m\left(B_{c}\right)}{p_{T}(J / \psi \mu)}$ where $L_{x y}$ is the transverse decay length. A correction factor based on MC is then applied to estimate the $p_{T}$ of the $B_{c}$ to determine the proper time. Fig 7 shows the visible proper decay length for the $J / \psi+\mu$ sample with projections of the fit superimposed. A lifetime of $\tau\left(B_{c}\right)=0.448_{-0.036}^{+0.038}$ stat \pm 0.032 (sys) ps is obtained, currently making this measurement the most precise measurement of the $B_{c}$ lifetime.

\section{4. $B_{s}$ mixing}

A measurement of the $B_{s}-\bar{B}_{s}$ oscillation frequency is a powerful test for the CKM formulism of the Standard Model. The CDF experiment has reported the oscillation frequency $\Delta m_{s}$ to be $17.77 \pm 0.10$ (stat) \pm 0.07 (sys) [4].

Using $2.8 \mathrm{fb}^{-1}$ of data, we have updated our oscillation frequency measurement. This measurement combines all of our semileptonic decay modes as well as the hadronic mode.

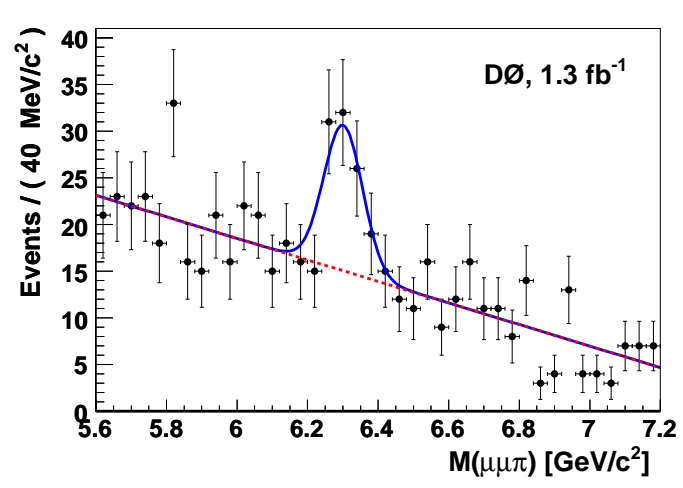

Figure 5. $J / \psi \pi$ invariant mass distribution. A projection of the unbinned fit is overlaid on the plot.

The data are fit using the amplitude fit method [5]. The values of $\Delta m_{s}$ are varied from 0 to 30 $\mathrm{ps}^{-1}$ in step sizes of $0.5 \mathrm{ps}^{-1}$. The fitted value of the amplitude $A$ is plotted as function of $\Delta m_{s}$. Fig. 8 shows the amplitude as function of $\Delta m_{s}$.

We plot the difference $\Delta \log L\left(\Delta m_{s}\right)=$ $\log L_{\Delta m_{s}}(A=1)-\log \left(L_{\infty}\right)$, see Fig. 9. The points are fitted to a parabolic function and a value of $\Delta m_{s}=18.56 \pm 0.87$ is obtained. The statistical significance of the minimum is $3.1 \sigma$.

5. $B^{+} \rightarrow J / \psi K^{+}$

The Standard Model predicts a very small charge asymmetry for $B^{ \pm} \rightarrow J / \psi K^{ \pm}$decays, where the charge asymmetry is defined as

$$
\begin{gathered}
A_{C P}\left(B^{+} \rightarrow J / \psi K^{+}\right)= \\
\frac{N\left(B^{-} \rightarrow J / \psi K^{-}\right)-N\left(B^{+} \rightarrow J / \psi K^{+}\right)}{N\left(B^{-} \rightarrow J / \psi K^{-}\right)+N\left(B^{+} \rightarrow J / \psi K^{+}\right)}
\end{gathered}
$$

A measurement of the charge asymmetry can therefore constrain those models which predict large asymmetries for $B^{ \pm} \rightarrow J / \psi K^{ \pm}$decays.

$B^{+}$decaying to $J / \psi K^{+}$events are reconstructed and shown in Fig. 10. This distribution is a combination of $B \rightarrow J / \psi K, B \rightarrow J / \psi \pi$, and $B \rightarrow J / \psi K^{*}$. Using an unbinned likelihood fit, 


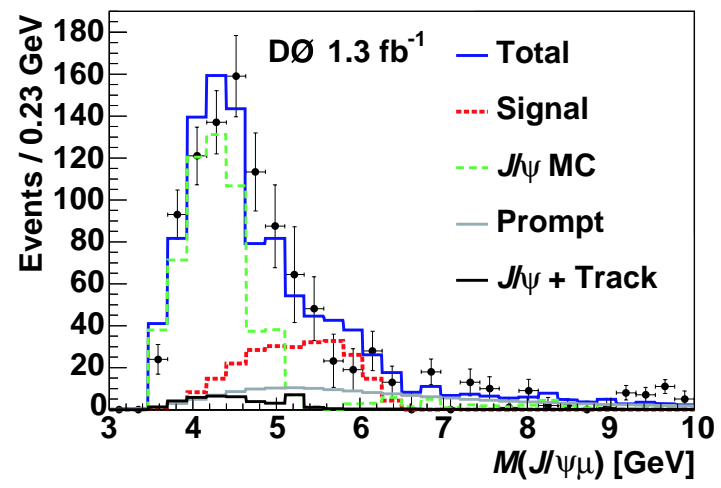

Figure 6. Fit to the $J / \psi+\mu$ mass with the $J / \psi$ mass sideband and $B^{+}$background subtracted. A decay length significance of greater than 4 has been applied.

the fraction of each decay is determined. The asymmetry due to physics and detector effects must be removed. In order to measure these asymmetries, the data are subdivided into eight samples with different solenoid and toroid polarities and different pseudorapidity of the $J / \psi \mathrm{K}$ system and charge of the kaon candidate. Fitting these eight samples allows an extraction of the true asymmetry. The direct $\mathrm{CP}$-violating charge asymmetry is measured to be $A_{C P}\left(B^{+} \rightarrow\right.$ $\left.J / \psi K^{+}\right)=+0.0075 \pm 0.0061($ stat $) \pm 0.0030($ syst $)$, making this the most precise measurement of this asymmetry.

\section{Upsilon polarization}

Non-relativistic QCD (NRQCD) is able to predict both the production and decay of quarkonium [6]. One of the predictions from NRQCD for quarkonium produced at the Tevatron is that the S-wave should be transversely polarized at high $p_{T}$. By measuring the polarization of the $\Upsilon(1 S)$ and $\Upsilon(2 S)$ at D0, this prediction can be tested.

One measure of the polarization is

$$
\alpha=\left(\sigma_{T}-2 \sigma_{L}\right) /\left(\sigma_{T}+2 \sigma_{L}\right)
$$

where $\sigma_{T}$ and $\sigma_{L}$ are the transversely and longi-

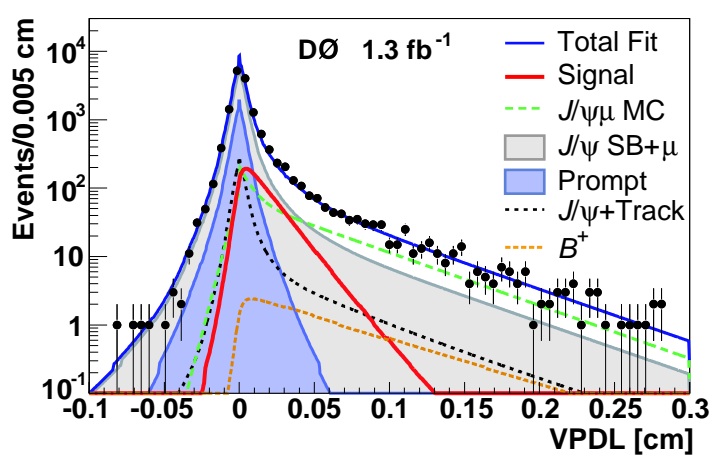

Figure 7. Visible proper decay length of the $J / \psi \mu$ sample with the fit projections overlaid.

tudinally polarized components of the production cross section. The angular distribution of quarkonium decaying into two muons is given by

$$
\frac{d N}{d\left(\cos \left(\theta^{*}\right)\right)} \propto 1+\alpha \cos ^{2}\left(\theta^{*}\right)
$$

where $\theta^{*}$ is the angle of the positively charge muon in the quarkonium center-of-mass frame with respect to the momentum of the $\Upsilon$ in the lab frame.

The dimuon invariant mass spectrum is fitted in bins of $\left|\cos \left(\theta^{*}\right)\right|$. Fig. 11 shows a fit to the invariant mass distribution for a particular $\left|\cos \left(\theta^{*}\right)\right|$ bin. The Monte Carlo does not accurately describe the data, so the MC was weighted so that both $p_{T}^{\Upsilon}$ and $p^{\Upsilon}$ agree. After this reweighting, there is good agreement between data and Monte Carlo, see Fig. 12.

Fig. 13 compares the measured values from D0 (circles) of $\alpha$ for the $\Upsilon(1 S)$ as a function of $p_{T}^{\Upsilon}$. Also shown in Fig. 13 are the prediction of NRQCD (grey band), and the two limits of the $k_{t}$ factorization model [7] (lower curve: quark-spin conservation hypothesis) (upper curve: full quark-spin depolarization hypothesis). Also shown (triangles) is the previous measurement from CDF [8]. Fig 14 compares the measured values from D0 (circles) of $\alpha$ for the $\Upsilon(2 S)$ as a function of $p_{T}^{\Upsilon}$ along with the predic- 


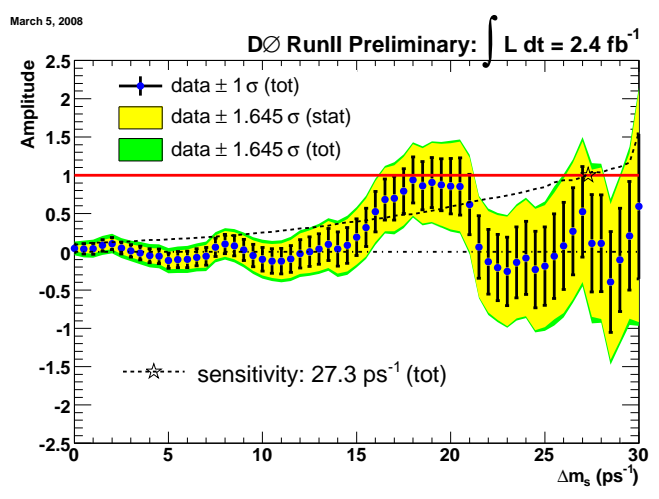

Figure 8. Combined $B_{s}$ oscillation amplitude as a function of $\Delta m_{s}$

tion of NRQCD (grey band).

Significant $p_{T}$ dependence is seen in the longitudinal polarization for the $\Upsilon(1 S)$ which is not seen in the NRQCD prediction.

\section{REFERENCES}

1. D. Acosta, et al. (CDF collaboration), Phys. Rev. Lett. 97, 242003 (2006)

2. W.-M. Yao et al., Journal of Physics G 33, 1 (2006).

3. R. Aleksan et. al., Phys. Lett. B, 316, 567 (1993).

4. A. Abulencia, et. al., Phys. Rev. Lett. 97 (2006) 242003.

5. H.G Moser, A. Roussarie, Nucl. Instrum. Meth. A384:491-505 (1997)

6. G.T. Bodwin, E. Braaten and G.P. Lepage. Phys. Rev. D 51, 1125 (1995) [Erratum-ibid. D 55, 5853 (1997)].

7. S.P. Baranov and N.P. Zotov, Pis'ma v ZhETF 86, 499 (2007).

8. D. Acosta, et al. (CDF collaboration), Phys. Rev. Lett. 88, 161802 (2002)

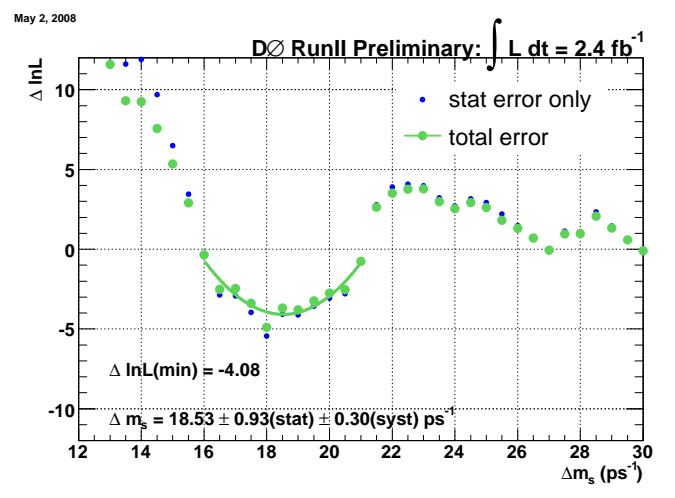

Figure 9. Combined dependence of $\Delta(\log L)$ on $\Delta m_{s}$.

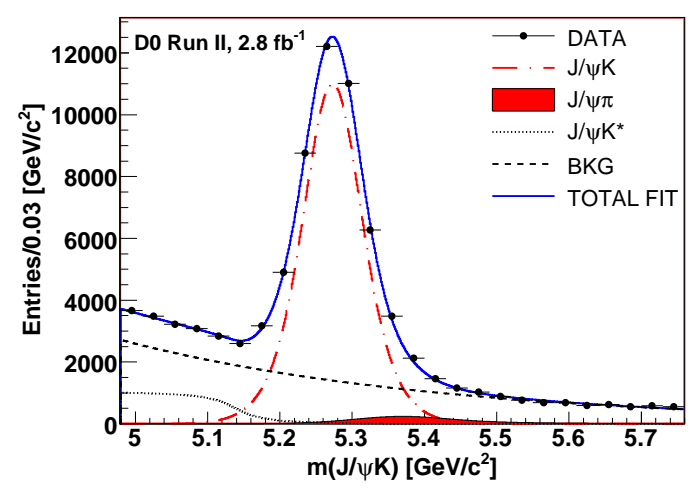

Figure 10. The $J / \psi K^{+}$invariant mass distribution along with the projection of the unbinned likelihood fit. 

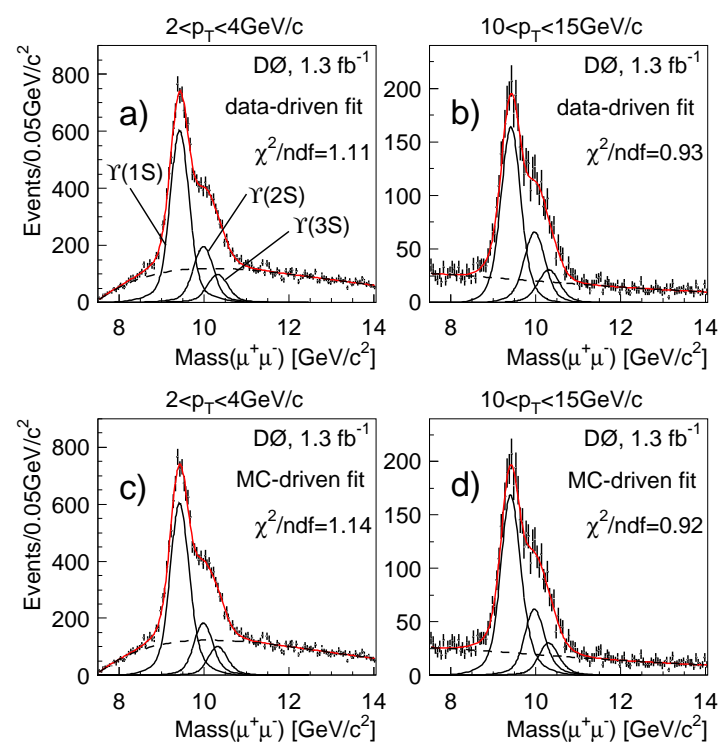

Figure 11. Dimuon invariant mass distribution for events in $0.4<\left|\cos \left(\theta^{*}\right)\right|<0.5$.
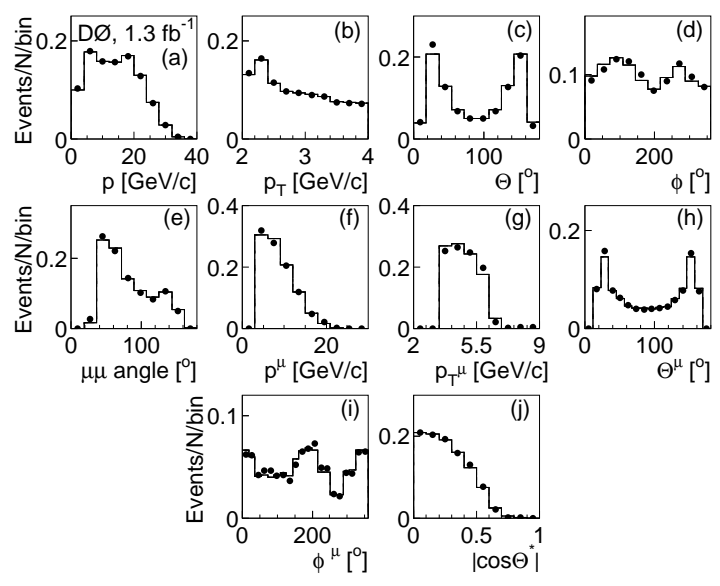

Figure 12. Comparison between Monte Carlo (solid histogram) and data(points) for $\Upsilon(1 S)$ with $2<p_{T}^{\Upsilon}<4 \mathrm{GeV}$.

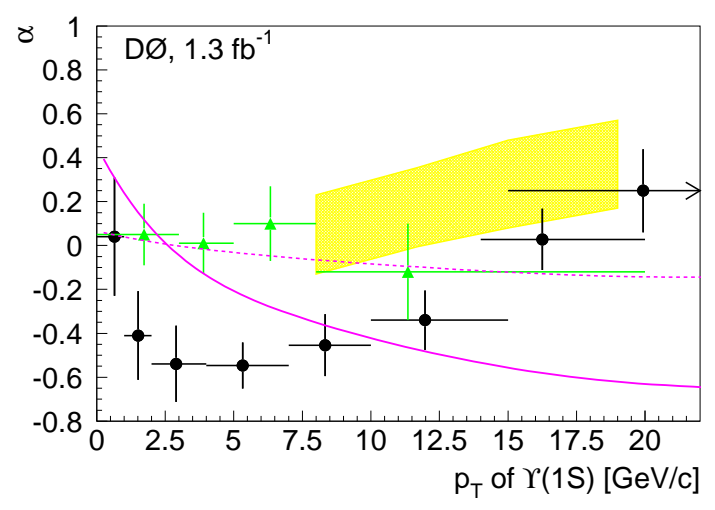

Figure 13. $\alpha$ dependence as a function of $p_{T}^{\Upsilon}$ for $\Upsilon(1 S)$. The different points and curves are explained in the text.

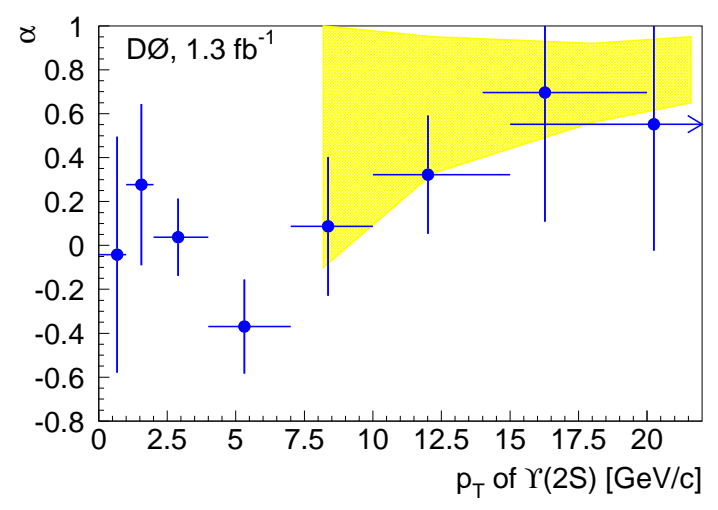

Figure 14. $\alpha$ dependence as a function of $p_{T}^{\Upsilon}$ for $\Upsilon(2 S)$. Circles are D0 data and the grey band is the prediction from NRQCD. 